\title{
"Let Food be Thy Medicine": Diet and Supplements in Irritable Bowel Syndrome
}

\author{
Neha V Patel, MD* \\ Digestive and Liver Diseases, Internal Medicine, University of Texas Southwestern Medical Center, USA
}

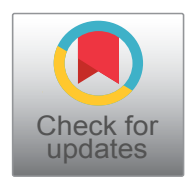

*Corresponding author: Neha V Patel, Digestive and Liver Diseases, Internal Medicine, University of Texas Southwestern Medical Center, 5959 HARRY HINES BLVD HP5.520, Dallas TX 75235, USA, Tel: 214-645-6355

\begin{abstract}
Overview: The purpose of this review is to introduce options of dietary therapies and supplements as treatments for the treatment of Irritable bowel syndrome (IBS). IBS is a common condition with heterogeneity in pathogenesis and clinical presentation. Current treatment options are targeted at symptom relief with medications. Dietary therapy for IBS has been poorly studied in the past, however newer evidence suggests the use of certain diets, such as the low FODMAP (Fermentable Oligosaccharides, Disaccharides, Monosaccharides and Polyols) diet, as an intervention in patients with IBS for symptom improvement. Other dietary strategies are frequently tried but lack quality evidence behind their use. Certain supplements, such as fiber, probiotics, and peppermint oil, have also been studied and are favored for use in IBS patients with specific caveats.
\end{abstract}

\section{Keywords}

IBS, FODMAP, Probiotics, Microbiome, Diarrhea, Constipation, Abdominal pain, Bloating, Gluten, Sensitivity, Dietary, Disease, Treatment

\section{Statement of Significance}

Dietary therapies and supplements have a role in the management of patients with Irritable Bowel Syndrome. Current review of the evidence supports use of the LowFODMAP diet and soluble fiber supplementation, and cautious use of probiotics in certain patients.

\section{Introduction}

Irritable bowel syndrome is a condition which is diagnosed based on symptoms defined by the Rome IV criteria. These criteria require the presence of abdominal pain and altered bowel habits. Symptoms should be present without a structural cause or any significant warning signs such as bleeding, weight loss or anemia, or nocturnal symptoms. Irritable bowel syndrome (IBS) is becoming increasingly common and costly. It is the most commonly diagnosed condition by gastroenterologists, affecting $11 \%$ of the world population and $12 \%$ of the US population. This condition equates to significant financial burden in healthcare, accounting for almost 6 million prescriptions and $\$ 20$ billion in healthcare-related costs. All-cause costs have been estimated annually at $\$ 11,182$ to $\$ 13,038$ per patient [1].

The pathogenesis of IBS is multifactorial and heterogeneous among patients with alterations in motility, visceral sensation, the microbiome, braingut interaction via the central and enteric nervous systems, mucosal immune function, bile acid metabolism and intestinal permeability. More recent studies have turned attention to the interplay between the microbiome, immune function, gut sensation and motility. The microbiome of IBS patients has a different luminal bacterial composition with fewer Bifidobacteria, and Faecalibacterium prausnitzii sp and more Lactobacillaceae, Bacteroides, Enterobacteriaceae $[2,3]$. These alterations in the microbiome have effects of increased visceral hypersensitivity with colonic distension in animal models. IBS patients have alterations in immune activation by increased toll-like receptor expression and production of proinflammatory cytokines. Degradation of tight junction proteins and increased intraepithelial permeability has been observed. This dysbiosis has effects on gut function and is an important player in the pathogenesis of IBS. 
Table 1: Current FDA-Approved Therapies for IBS.

\begin{tabular}{|l|l|l|l|l|l|}
\hline Drug Mechanism & $\begin{array}{l}\text { FDA-Approved } \\
\text { Drug }\end{array}$ & Indication & $\begin{array}{l}\text { Percentage } \\
\text { response drug }\end{array}$ & $\begin{array}{l}\text { Percentage } \\
\text { response placebo }\end{array}$ & $\begin{array}{l}\text { Number needed } \\
\text { to treat (NNT) }\end{array}$ \\
\hline Microbiome & Rifaximin & IBS-D & 43 & 34 & 11 \\
\hline Hypersensitivity and motility & Eluxadoline & IBS-D & 27 & 17 & 10 \\
\hline Motility & Alosetron & IBS-D & 51 & 36 & 7 \\
\hline \multirow{2}{*}{ Gut secretion } & Lubiprostone & IBS-C & 18 & 10 & 12.5 \\
\hline & Linaclotide & IBS-C & 34 & 14 & 5 \\
\hline & Plecanatide & IBS-C & 30 & 18 & 8 \\
\hline
\end{tabular}

IBS-D: Irritable Bowel Syndrome-Diarrhea predominant; IBS-C: Irritable Bowel Syndrome-Constipation predominant

\section{Current Status of Knowledge}

With the heterogeneity of symptoms and multiple postulated mechanisms of disease, therapeutic options for IBS have been disappointing. Current medications for IBS target certain mechanisms such as motility or secretion or the microbiome. In randomized controlled trials (RCTs), these medications offer therapeutic gains ranging from only $8-20 \%$ over placebo, making maybe less than $1 / 2$ of patients better (Table 1 ).

A significant portion of patients turn to selfdirected care or use "alternative" medicines for their symptoms. Nutritional components of food have been used medicinally for thousands of years. Hippocrates, the father of modern western medicine, was quoted: "Let food be thy medicine and medicine be thy food". Medicines derived from foods or plants have become more widely available especially with online shopping and increase in health food stores. These alternative therapies such as probiotics and supplements, or medical foods are not regulated by the Food and Drug Administration (FDA). They are not held to the rigorous standards in clinical trials, lending to variability among trial results. Current dietary studies have been largely of poor quality with small size, lack of blinding and randomization, and significant bias. More recently certain diets and supplements have gained some momentum in research studies in IBS.

\section{Fiber Supplementation}

Increase in dietary fiber has been recommended by physicians for IBS for decades. Fiber has effects on colonic secretions, motility, and the microbiome. Soluble fiber mixes with water creating a gel-like matter which is digested by bacteria and improves stool viscosity. Insoluble fiber increases stool volumes. Fiber supplementation has been adopted into practice, however studies on fiber use had poor methodology and high risk of bias. To date, there have been 15 RCTs of fiber use in IBS in 1048 patients which showed significant effect in global symptoms [4]. There was a statistically significant benefit for use of ispaghula or psyllium compared to placebo. Bran, which is an insoluble fiber, had no significant effect in IBS symptom relief. Soluble fibers such as psyllium or wheat dextran or acacia, is a reasonable low-cost intervention and recommended for patients with Irritable bowel syndrome with constipation.

\section{Probiotics, Prebiotics, and Synbiotics}

Alterations in the gut microbiome contribute to the pathogenesis of IBS, which is also supported by symptoms of IBS developing after an infection, known as post-infectious IBS. This raises the question whether using agents to alter the microbiome improves IBS symptoms. Probiotics, prebiotics, and synbiotics have emerged as agents which modify the microbiome for benefit to the host. Probiotics are live microorganisms which may confer health benefit. Prebiotics are the substrates which are utilized by the present gut microorganisms which essentially feed the microbiome. Synbiotics are a mixture of substrates (prebiotics and live organisms) which would act synergistically to promote the growth of certain beneficial organisms. There have been 18 new RCTs assessing efficacy of probiotics in IBS in the past 5 years alone [5]. Two RCTs of the prebiotic fructo-oligosaccharide versus placebo reported no significant difference in IBS symptoms with prebiotic use. One study evaluating a high-dose and low-dose prebiotic galacto-oligosaccharide showed significant improvement in overall symptoms and no change in abdominal pain. The two RCTs of synbiotic use in IBS were conducted in Italy and S Korea with both trials showing positive results without statistically significant effect in reducing symptoms. Based on the current evidence, probiotics are cautiously recommended for the improvement of symptoms, particularly bloating and flatulence, in IBS patients. There is not enough evidence to support the use of prebiotics or synbiotics. Probiotics come in many different varieties and are sold as combinations, tablet or liquids, and refrigerated forms. Combinations of probiotics, Lactobacillus $s p$, Bifidobacteria $s p$, and Streptococcus $s p$ confer beneficial effects in IBS, yet which specific combination or species still remains unclear.

\section{Peppermint Oil}

Peppermint oil has been used for its health benefits for many years, dating back to the 1700s, and has been most studied in IBS. Peppermint oil has been shown 
to exert effects on smooth muscle calcium channels and opioid receptors leading to muscle relaxation, changes in contractions or motility, and alteration of pain sensitivity. A systematic review and meta-analysis of five randomized controlled trials of peppermint oil (197 patients on peppermint oil and 195 on placebo) favored its use over placebo (RR $2.23(95 \% \mathrm{Cl} 1.78$ to 2.81)) [6]. Global improvement of IBS symptoms and improvement in abdominal pain was seen in patients receiving peppermint oil compared to placebo. These effects on pain improvement are quick-acting, modest, and typically short-lived. Peppermint oil is recommended for use in IBS patients for pain, with the caveat that the data is based on few small studies with specific formulations of the supplement.

\section{Dietary Therapy for IBS}

The vast majority of IBS patients perceive their symptoms as related to either specific foods or meal intake in general [7]. While many patients jump to the conclusion that this implies a food allergy, there are many potential explanations for such postprandial exacerbations of symptoms, most of which represent activation of physiologic rather than allergic responses. Foods with high content of fat and spice are thought to give rise to Gl symptoms. When surveying 197 Swedish patients with IBS about foods as triggers for their symptoms, the culprit foods were those containing carbohydrates and histamine. Contrary to popular belief, fatty foods were less commonly identified as triggers. Histamine and other biogenic amines occur in fish, fermented foods, vegetables, wine and beer. High levels of histamines can provoke symptoms such as nausea, abdominal pain and diarrhea. Alternatively, foods may not contain a significant amount of histamine but may trigger an endogenous histamine release. Still patients cannot help relating their symptoms to food intake and continue to search for triggers. They are trying different strategies to manage their symptoms and doing this without any medical guidance. A survey of 1500 Gastroenterologists across the US found that $50 \%$ of patients try a form of exclusion diet based on food triggers, and a $1 / 4$ of patients have tried a glutenfree diet (GFD) [8]. Dietary modification is ubiquitous and commonplace in clinical practice.

\section{Exclusion Diets Based on Testing}

Many patients keep food diaries or trial exclusion diets based on triggers. When rechallenged with the foods they perceive as triggers, the majority of patients do not report exacerbation of symptoms. Certain commercially available tests are based on gut immune activation to specific foods and claim to identify food intolerances, however these tests are not validated. The tests can be quite costly (up to $\$ 1000$ per panel) with poor coverage by commercial providers. IgG-based food allergy panel testing is frequently requested by patients and performed with insufficient data to support this practice. Food-related IgG4 testing is also available, but only indicates repeated exposure to foods, and does not indicate allergy, intolerance, nor hypersensitivity. IgG or IgG4 Food-allergy testing is not recommended by the GI and Allergy societies. Exclusion diet based on leukocyte antibody testing (LAT) of peripheral blood or the ALCAT test was studied in one trial in IBS patients. Fifty-eight patients randomized to an exclusion diet based on LAT or sham diet reported improvement in symptoms, however no significant difference was seen in overall quality of life and adequate relief of symptoms [9]. These results spark interest in immune activation in IBS and larger studies are needed.

\section{Lactose Restriction}

Many IBS patients attribute their symptoms to milk or dairy intolerance. This self-reported intolerance does not correlate with objective evidence of malabsorption, such as hydrogen breath testing. Older poor-quality trials reported robust symptom response to lactose restriction. This response may be a consequence of lactase deficiency, yet a study providing lactase supplementation to IBS patients did not show improvement of their symptoms [10]. There are no blinded or controlled trials which show benefit for lactose restriction. Hence there is no high-quality evidence to support use of lactose restriction in IBS patients.

\section{Gluten Restriction}

Some patients attribute their IBS symptoms to gluten or wheat ingestion. Attention to glutenrelated conditions, including Celiac Disease and gluten intolerance or allergy, has increased dramatically, reflecting the marked growth of the glutenfree food industry. This has extended to the population of healthy individuals who believe that adhering to a GFD may have benefits on overall health regardless of Gl symptoms. The prevalence of people who avoid gluten has more than tripled from 2009 to 2014, with nearly 3 million people following a GFD. There are two well-recognized clinical entities where GI symptoms are triggered by gluten, which may present with symptoms similar to IBS. Celiac Disease (CD) is an enteropathy where gluten exposure to genetically predisposed individuals with HLA DQ2 or DQ8 genotype results in inflammation characterized by infiltration of the lamina propria and epithelium with lymphocytes and villous atrophy. Patients have elevated serum biomarkers and may have extra-intestinal manifestations such as a rash called dermatitis herpetiformis or vitamin deficiencies or osteoporosis. Non-celiac gluten sensitivity (NCGS) is an entity where patients have symptoms related to gluten intake and improve on a gluten-free diet without any genetic predisposition or serum biomarker elevation or enteritis. The pathogenesis of NCGS has some hallmarks 
of innate immune system activation, however this has not been clearly defined. Since patients with Celiac Disease and NCGS present with symptoms paralleling IBS, it is of utmost importance to exclude CD due to long-term implications such as malignancy, infertility, and osteoporosis. What is alarming though is that $62 \%$ of adults who report an intolerance to gluten have not had appropriate biomarkers and testing for $C D$ before initiating a gluten-free diet.

Gluten-free diets may help for certain IBS patients. A double-blinded RCT exposed patients without Celiac Disease who previously responded to gluten-free diet to either gluten or placebo diets. Of the 19 patients in the gluten arm, $68 \%$ of patients reported uncontrolled symptoms compared to $40 \%$ of patients in the placebo arm. Patients exposed to gluten complained of worsening pain, bloating, stool consistency, and fatigue [11]. While other trials suggest improved outcomes for patients on gluten-free diet, there are only two small randomized controlled trials assessing gluten-free diet in IBS involving 111 participants. Both trials reported statistically significant results, however in a pooled analysis, the results were not statistically significant [12]. Perhaps a more personalized approach may increase the efficacy of gluten restriction in IBS patients. One study explored the effects of gluten-free or gluten-containing diets on patients with diarrhea-predominant IBS. A gluten-containing diet was associated with increased bowel movements and small bowel permeability, especially in those positive for HLA DQ8 or DQ2 [13]. Patients with diarrhea-predominant symptoms may have a better response. Another explanation for the unconvincing evidence for gluten-free diet is perhaps the issue is not gluten alone but the other components of wheat products causing symptoms. Fructans are a polymer of fructose molecules that are present in many foods and grains including wheat, barley, rye, onions, and garlic. Wheat also contains other proteins called amylase trypsin inhibitors which can exacerbate GI symptoms. To test this hypothesis, a study from Norway took 59 patients with self-reported gluten sensitivity and randomized them to fructan diet, gluten diet, or placebo for one week [12]. Results demonstrated significantly higher symptom scores after fructan intake rather than gluten intake or placebo. The problem may not be gluten specifically, but rather the fructans in wheat which lead to symptoms.

\section{Low FODMAP Diet}

A group in Australia developed a diet that focused on lowering the amount of poorly absorbed, fermentable carbohydrates collectively, called the low FODMAP diet.

FODMAPs are fermentable oligo-, di-, and monosaccharides and polyols. Oligosaccharides include fructans such as onions, shallots, garlic, barley, cabbage, artichoke, asparagus and galacto-oligosaccharides like beans and chickpeas. Foods high in monosaccharides like fructose include apples, pears, watermelon, and mango. Examples of polyols are sorbitol, mannitol, and other artificial sweeteners. FODMAPs are characterized by limited small intestinal absorption and are broken down by bacteria to short chain fatty acids (SCFAs). SCFAs create high osmotic activity which increases intestinal secretions and water content and lowers luminal $\mathrm{pH}$. In healthy individuals, functional MRI testing after ingesting fructose showed increased small bowel water content [14]. Ingestion of fructose and inulin, which is a fructan, also led to increased hydrogen breath production and increased colonic luminal distension. These effects rarely cause symptoms in healthy subjects. When coupled with visceral hypersensitivity in IBS patients, these effects have the capability of inducing significant GI symptoms. High FODMAP foods exert effects on the colon through bacterial fermentation which produces higher levels of hydrogen and methane gas. IBS patients on high FODMAP diets have been shown to produce higher levels of breath hydrogen than healthy volunteers and higher breath hydrogen levels than IBS

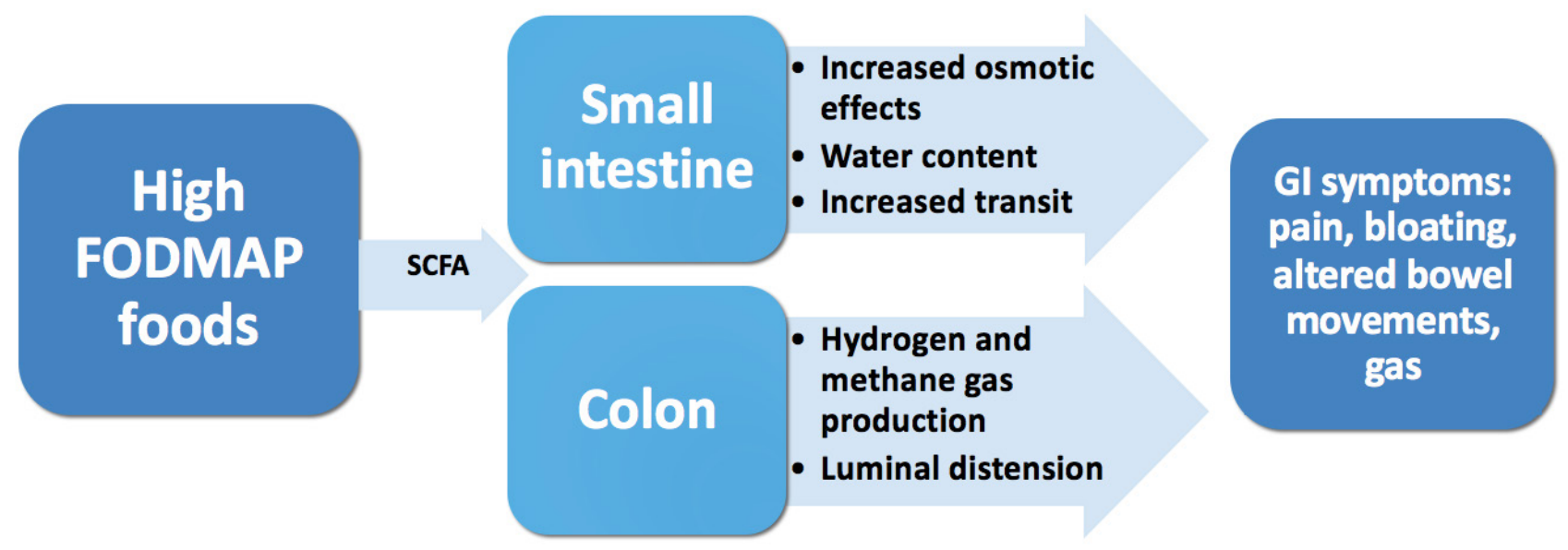

Figure 1: FODMAP effects on the small intestine and colon lead to symptoms.

FODMAP: Fermentable Oligosaccharides, Disaccharides, Monosaccharides and Polyols; SCFA: Short-Chain Fatty Acids; GI: Gastrointestinal 
patients on a low FODMAP diet [15]. FODMAPs induce effects on the small intestine and colon which lead to pain, gas/bloating, and altered bowel movements in IBS patients (Figure 1).

There is growing data which supports the use of the low FODMAP diet (LFD) in IBS. Seven randomized controlled trials evaluated 397 participants on a low FODMAP diet compared to either an alternate sham or placebo diet, an IBS diet based on NICE guidelines, or high FODMAP diet. The low FODMAP diet was effective in improving IBS symptoms with a RR of continued symptoms on a low FODMAP diet of $0.69(95 \% \mathrm{Cl} 0.54$ to 0.88$)$. The NNT for the FODMAP diet was $5(95 \% \mathrm{Cl}$ 3 to 11) [16]. An earlier randomized controlled trial compared the FODMAP diet to placebo and provided all participants with their meals. Symptom scores and stool samples were analyzed of 30 patients and 8 controls randomized to LFD or typical Australian diet for 21 days. IBS patients on LFD had lower overall symptom scores than patients on typical Australian diet. Individual symptoms of pain, bloating, and flatulence were also improved. There was no significant difference in fecal indices such as stool weight, frequency, water content, and form [17]. More recently, a study compared the effects of a low FODMAP diet to an IBS diet based on NICE (National Institute for Health and Care) guidelines in patients with diarrhea-predominant symptoms. There was a higher number of abdominal pain responders in the LFD group compared to the NICE diet (51\% vs. $23 \%, P=0.008$ ) [18]. Though there was no significant difference in adequate relief of symptoms or the composite end point between the groups. Compared with baseline scores, the low FODMAP diet led to greater reductions in average daily scores of abdominal pain, bloating, consistency, frequency, and urgency. More studies report IBS symptom improvement on the Iow FODMAP diet.

The diet is not just eliminating high FODMAP foods. It begins with an elimination period for 4-6 weeks which is viewed as a diagnostic test to identify patients sensitive to FODMAPs. Those who fail to improve do not continue the diet. Those with response should be instructed on reintroducing foods which contain FODMAPs gradually to identify their sensitivities. This information should then be used to liberalize and personalize their diet to minimize symptoms. The FODMAP diet can limit calcium intake significantly to less than daily recommended intake $(<800 \mathrm{mg} / \mathrm{d})$ during the elimination phase [19]. It also limits fiber intake. While there may be slight differences in micronutrient intake, such as thiamine and riboflavin, during the diet, these are still within normal range and not significant clinically.

The FODMAP diet also has the ability to alter immune regulation in the gut. Hustoft and colleagues reported improved IBS symptoms and decreased proinflammatory cytokines IL-6 and IL- 8 in their double- blinded RCT of LFD [20]. In another study, urinary histamine, a marker of immune activation, decreased 8 -fold in those on the low FODMAP diet [21]. The diet alters the microbiome with reduction in concentration of species and a decrease in total bacteria. Certain beneficial species, Bifidobacteria, Actinobacteria and Faecalibacterium prausnitzii, were less abundant in IBS patients on the low FODMAP diet, which some speculate to be a result of reducing dietary prebiotic components. These effects are short-lived as several of these bacteria rebounded after 10 days of high-fructooligosaccharide (high FODMAP) supplementation [20].

Currently there are no identifiable characteristics that predict response to the diet. A Norwegian study analyzed gut microbial composition as a marker for the response to FODMAP diet by assessing 54 fecal bacterial markers with 16s rRNA sequencing. Responders had higher level of markers for Bacteroides fragilis, Acinetobacter, Ruminiclostridium, Streptococcus and Eubacterium and lower levels of markers for Clostridia, Actinomycetes, and Shigella/Escherichia [22]. Another study measured volatile organic compounds in gases emanated from stool in patients on a low FODMAP diet. Results showed certain patterns of volatile organic compounds could identify patients who are responders to the low-FODMAP diet or a probiotic supplement with a mean accuracy of $97 \%$ and $89 \%$ respectively [23]. Perhaps gut microbiota composition or VOC profiling may develop as tools to identify response to dietary FODMAP restriction, however further studies are necessary.

Implementation and adherence to the diet can be an arduous task for many patients. Removal of dairy products, wheat products, certain fruits and vegetables and sweeteners can make food options quite limited. Physicians lack the time and sometimes the expertise in counseling patients on diet modifications. Referral to a dietician is recommended when feasible. If the dietician visit is not covered by insurance, patients can use online support and even apps such as the Monash FODMAP app to identify foods. Recipe books and blogs by registered dieticians are available online which can guide patients on meal preparation. The FODMAP diet has been viewed by gastroenterologists as a primary strategy for therapy in IBS. Many GIs consider the diet as effective, if not better, than current medical treatments. The American College of Gastroenterology has recommended a low FODMAP diet for a limited time in the guidelines for management of IBS for overall symptom improvement based on the current available data. They recommended against the use of gluten-free diet or exclusion diets based on antibody or leukocyte activation testing [16]. Personalized dietary modification, i.e. gluten or wheat avoidance or diet based on testing, still needs further study. How to identify patients with higher likelihood of response to dietary therapy is also an area of growing interest. Dietary strategies for the management of IBS 
are becoming more evidence-based than in earlier practice and a primary strategy for therapy in IBS, similar to other chronic disease conditions such as diabetes or cardiovascular disease.

\section{Conflict of Interest}

This is to acknowledge that Neha Patel, MD has disclosed that she does not have any financial interests or other relationships with commercial concerns related directly or indirectly to this journal.

\section{Sources of Funding Support}

None.

\section{References}

1. Jessica L Buono, Kush Mathur, Amelia J Averitt, David A Andrae (2017) Economic burden of irritable bowel syndrome with diarrhea: Retrospective analysis of a u.s. commercially insured population. J Manag Care Spec Pharm 23: 453460.

2. Hai-Ning Liu, Hao Wu, Yu-Zhuo Chen, Yan-Jie Chen, XiZhong Shen, et al. (2017) Altered molecular signature of intestinal microbiota in irritable bowel syndrome patients compared with healthy controls: A systematic review and meta-analysis. Dig Liver Dis 49: 331-337.

3. Rapat Pittayanon, Jennifer T Lau, Yuhong Yuan, Grigorios I Leontiadis, Frances Tse, et al. (2019) Gut microbiota in Patients with irritable bowel syndrome-a systematic review. Gastroenterology 157: 97-108.

4. Neeraja Nagarajan, Amanda Morden, Danielle Bischof, Elizabeth A King, Martin Kosztowski, et al. (2015) The role of fiber supplementation in the treatment of irritable bowel syndrome: A systematic review and meta-analysis. Eur $\mathrm{J}$ Gastroenterol Hepatol 27: 1002-1010.

5. Alexander C Ford, Lucinda A Harris, Brian E Lacy, Eamonn M M Quigley, Paul Moayyedi, et al. (2018) Systematic review with meta-analysis: The efficacy of prebiotics, probiotics, synbiotics and antibiotics in irritable bowel syndrome. Aliment Pharmacol Ther 48: 1044-1060.

6. Reena Khanna, John K MacDonald, Barrett G Levesque (2014) Peppermint oil for the treatment of irritable bowel syndrome: A systematic review and meta-analysis. J Clin Gastroenterol 48: 505-512.

7. Lena Böhn, Stine Störsrud, Therese Liljebo, Lena Collin, Perjohan Lindfors, et al. (2015) Diet low in FODMAPs reduces symptoms of irritable bowel syndrome as well as traditional dietary advice: A randomized controlled trial. Gastroenterology 149: 1399-1407.

8. Adrienne Lenhart, Courtney Ferch, Michael Shaw, William D Chey (2018) Use of dietary management in irritable bowel syndrome: Results of a survey of over 1500 united states gastroenterologists. J Neurogastroenterol Motil 24: 437-451.

9. Ather Ali, Theresa R Weiss, Douglas McKee, et al. (2017) Efficacy of individualised diets in patients with irritable bowel syndrome: A randomised controlled trial. BMJ Open Gastroenterol 4: 000164.

10. R Lisker, NW Solomons, R Pérez Briceño, M Ramírez Mata (1989) Lactase and placebo in the management of the irritable bowel syndrome: A double-blind, cross-over study. Am J Gastroenterol 84: 756-762.
11. Jessica R Biesiekierski, Evan D Newnham, Peter M Irving, et al. (2011) Gluten causes gastrointestinal symptoms in subjects without celiac disease: A double-blind randomized placebo-controlled trial. Am J Gastroenterol 106: 508-514.

12. Joanna Dionne, Alexander C Ford, Yuhong Yuan, Jacqueline S Barrett, Melissa Haines, et al. (2018) A systematic review and meta-analysis evaluating the efficacy of a gluten-free diet and a low FODMAPs diet in treating symptoms of irritable bowel syndrome. Am J Gastroenterol 113: $1290-1300$.

13. Maria Vazquez-Roque, Michae Camilleri, Thomas Smyrk, Joseph A Murray, Eric Marietta, et al. (2013) A controlled trial of gluten-free diet in patients with irritable bowel syndrome-diarrhea: Effects on bowel frequency and intestinal function. Gastroenterology 144: 903-911.

14. Kathryn Murray,VictoriaWilkinson-Smith,Caroline Hoad, Hoad Caroline, Costigan Carolyn, et al. (2014) Differential effects of FODMAPs fermentable oligo-, di-, monosaccharides and polyols on small and large intestinal contents in healthy subjects shown by MRI. Am J Gastroenterol. 109: 110-119.

15. Derrick K On, Shaylyn B Mitchell, Jacqueline S Barrett, Sue J Shepherd, Peter M Irving, et al. (2010) Manipulation of dietary short chain carbohydrates alters the pattern of gas production and genesis of symptoms in irritable bowel syndrome. J Gastroenterol Hepatol 25: 1366-1373.

16. Alexander C Ford, Paul Moayyedi, William D Chey, Lucinda A Harris, Brian E Lacy, et al. (2018) American college of gastroenterology monograph on management of irritable bowel syndrome. Am J Gastroenterol 113: 1-18.

17. Emma P Halmos, Victoria A Power, Susan J Shepherd, Peter R Gibson, Jane G Muir (2014) A diet low in FODMAPs reduces symptoms of irritable bowel syndrome. Gastroenterology 146: 67-75.

18. Shanti L Eswaran, William D Chey, Theresa Han-Markey, Sarah Ball, Kenya Jackson (2016) A randomized controlled trial comparing the low FODMAP diet vs. modified NICE guidelines in US adults with IBS-D. Am J Gastroenterol 111: 1824-1832.

19. Shanti Eswaran, Russell D Dolan, Sarah C Ball, Kenya Jackson, William Chey, et al. (2019) The Impact of a 4-Week Low-FODMAP and mNICE Diet on Nutrient Intake in a Sample of US Adults with Irritable Bowel Syndrome with Diarrhea. J Acad Nutr Diet 120: 641-649.

20. TN Hustoft, T Hausken, SO Ystad, J Valeur, K Brokstad, et al. (2017) Effects of varying dietary content of fermentable short-chain carbohydrates on symptoms, fecal microenvironment, and cytokine profiles in patients with irritable bowel syndrome. Neurogastroenterol Motil 29.

21. Keith Mclntosh, David E Reed ,Theresa Schneider, Frances Dang, Ammar H Keshteli, et al. (2017) FODMAPs alter symptoms and the metabolome of patients with IBS: A randomised controlled trial. Gut 66: 1241-1251.

22. Jørgen Valeur, Milada Cvancarova Småstuen, Torunn Knudsen, Gülen Arslan Lied, Arne Gustav Røseth (2018) Exploring gut microbiota composition as an indicator of clinical response to dietary FODMAP restriction in patients with irritable bowel syndrome. Dig Dis Sci 63: 429-436.

23. Megan Rossi, Raphael Aggio, Heidi M Staudacher, Miranda C Lomer, James O Lindsay, et al. (2018) Volatile organic compounds in feces associate with response to dietary intervention in patients with irritable bowel syndrome. Clin Gastroenterol Hepatol 16: 385-391. 